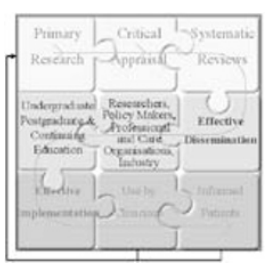

\title{
Understanding the jigsaw of evidence-based dentistry. 2. Dissemination of research results
}

\section{Nigel Pitts}

Director, Dental Health Services Research Unit and Centre for Clinical Innovations, University of Dundee, Dundee, UK

The first part of this three-part series provided an overview and a definition of evidence-based dentistry (EBD). ${ }^{1}$ Having introduced the EBD matrix, part one concentrated on the research synthesis part of the jigsaw puzzle. Now we focus on the middle row of this puzzle, the dissemination of research results. The final part of this series, to be published in a forthcoming issue, will consider the bottom row of the EBD matrix, the implementation of research findings in clinical practice.

Evidence-Based Dentistry (2004) 5, 33-35. doi:10.1038/sj.ebd.6400256
The realisation that evidence frequently takes years to influence clinical practice or, worse, might never do so, means that expectations are changing. It is no longer sufficient for a scientist to carry out a meticulous study, secure publication in a scientific journal, and hope that clinicians and others may happen upon his/ her findings and then be swayed by the intellectual argument. It is now recognised that, ideally, all robust new evidence should be reliably, rapidly and systematically conveyed to individual clinicians and their teams, and thus contribute to the planning of individualised and appropriate care for patients. This responsibility and the clinical judgements for the planning of care remains with the dentist and his or her team. EBD seeks to inform these judgements, which must also take into account all the circumstances and preferences specific to the individual patient concerned.

\section{Effective dissemination}

Figure 1 shows the middle row of the EBD matrix. If we look first at the righthand side, it is evident that we need not just dissemination of research findings, but effective dissemination. The previously accepted assumption that research published in a journal or presented at a learned scientific meeting would then somehow come to professional notice, gain wide acceptance and later be adopted into clinical practice by the majority has been shown to be something of a mirage. In reality, the communication of research findings was (and often still is) a haphazard, variable, unpredictable and unreliable process. Merely publishing in journals has been shown to be a largely ineffective strategy. $^{2}$ Most clinicians do not have sufficient time (or the facilities) to access and read a number of different journals regularly in the hope that they will discover individual articles of direct use to them.

A further problem is that, just when the proponents of EBD wish clinicians to be accessing research findings more often, there is a dramatically increasing volume of variable-quality research to sift through. There is also an increasing body of knowledge indicating that health professionals do not regularly obtain clinically useful material from published journal articles. ${ }^{2}$ It appears that research findings need to be disseminated in a more active way - to be marketed to different groups efficiently and in attractive formats that are accessible to the practising dentist. Unfortunately, in dentistry (as in other fields) very little attention has so far been paid to this task. Nor has there been much success in finding resources for this neglected task. In the UK there is now an opportunity to move forward, with the plans for the virtual Centre for Improving Oral Health $^{3}$ and the increasing education requirements for continuing professional development as well as the communication possibilities of the revolution in information technology.

The culture of the research community has been to focus on scientific publication in peer-review journals and this has been intensified further by the series of Research Assessment Exercises (RAEs) in the UK and equivalent research quality audits elsewhere. It is to be hoped that the modifications to the RAE that increase the emphasis on applied research will help redress the balance.

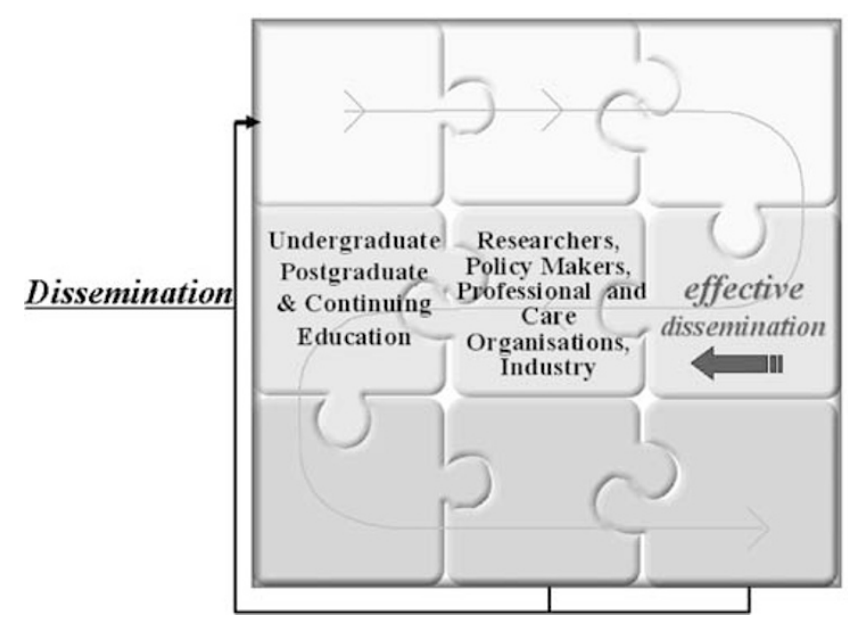

Figure 1. How dissemination should contribute to effective dental care with reference to the evidence-based dentistry matrix (EBD matrix figures (c) NB Pitts). 
Having achieved publication in an appropriate journal, the researcher often feels that his/her task has been accomplished; others contend that the scientist then has a responsibility to promote the communication of their findings. A further tension in many countries is that researchers can be pressured into publishing only in journals that have high impact factors. These are often inaccessible for the majority in research and for almost all in clinical practice. Publication in lesser journals, or even magazines that might reach end-users of the research findings more readily, is frowned upon in this environment. Nevertheless, this may be a more effective way of communicating research findings. A relatively simple way of increasing access to published research findings is to provide searchable reference lists on websites (see www.dundee.ac.uk/dhsru/) and to use practitionerfriendly, online journals (www.dundee. ac.uk/tuith/). It is acknowledged that such electronic resources for dissemination will currently appeal only to a subset of dentists and that a range of other strategies need to be developed and tested.

Historically, many research funders and employers have not been keen to pay for dissemination activities, even though it is likely to produce a better return on their investment. A related difficulty is that some excellent researchers are not equipped with the communication skills to disseminate their findings to clinicians, the media, or even to colleagues. Nor do many have the time, resources or opportunity for training in this area. In some cases, responsible marketing organisations may be better equipped to undertake such tasks.

It is frequently not clear who should be responsible for dissemination; that is, should this task be the obligation of the researcher, the research funder, or the health service/organisation concerned? As these issues will differ for different studies and types of research/setting, this should be agreed prospectively on a caseby-case basis. There is a growing trend for research grants to require applicants to outline planned dissemination activities and to stipulate this as a condition of funding. Although effective dissemination is now an area of study in its own right, the choice of method(s) employed (eg, targeted professional journal articles, conference papers, CD-ROMs, Internet posting or inclusion in continuing pro- fessional development activities) is still largely empirical.

\section{Research, industry, dental care and professional organisations}

Now we reach the central square of the EBD matrix, a key element for sharing research findings with potential users eager to have and use them. Perversely, however, this cell contains a number of types of organisation that, to date, have not seen themselves as involved with the EBD process or who have been reluctant to take an active part in the process. This may change as a greater understanding spreads about the value and utility of EBD to a broader number of groups who share a common, ultimate, focus on improving patient care.

\section{Researchers}

Researchers have a key role in the EBD process and of course provide the core resource. Much more could be done to communicate to more of the diverse research community the full range of components of EBD and explain how they can contribute to a variety of cells of the EBD matrix. A key and synergistic linkage is effective communication between researchers and the providers of dental education. Although in the past many of the researchers also taught in dental schools, the development of basic science institutes and other financial pressures in many countries have led to an increasing physical and, in some cases, intellectual separation between researchers and dental teachers.

\section{Research associations}

The missions of most research associations mean that their aims are congruent with the aims of EBD. More associations are becoming aware of this and facilitating EBD activities for their members, from organising symposia to promoting more systematic reviews. An example is provided by the European Organisation for Caries Research (ORCA), whose aims have for many years mirrored key parts of EBD definitions. ${ }^{4}$ For its 50th anniversary, ORCA mounted a symposium to review the art and science of cariology. One outcome from several papers presented there was a recognition that there is a deficit in knowledge transfer from the strong evidence base - the basic science research in cariology showing the dynamic nature of the initial, reversible stages of caries - that has been established over the last 25 years. This evidence base offers real clinical choices, with the possibility of adopting more preventive treatment decisions and therapies, ${ }^{4}$ yet it is not common knowledge for dental practitioners, authorities and, in some cases, educators.

Another positive example of research organisations responding to the need for more active dissemination of research findings is the recent developments in the International Association for Dental Research (IADR). IADR has now progressed from an ad-hoc committee coordinating an informal international collaboration for EBD, to the creation of an IADR EBD network, the 'IADR EBDNet'. This was approved by the IADR Council at its 2004 meeting. The IADR EBD-Net's primary task will be to establish efficient, non-duplicating links across all IADR scientific groups, from the international dental research community (and the evidence base its members are producing). Using these links parties seeking to transfer research evidence into clinical practice (such as the FDI World Dental Federation with its rich network of national dental associations) can be updated.

Unfortunately, some other research associations focus exclusively on research matters. The internal politics of some parts of the research community do not seriously entertain the communication and use of research findings as part of their business.

\section{Research funders}

Organisations that fund dental research are increasingly adopting EBD methodology as the foundation of their activities. Examples include the National Institute for Dental and Cranio-facial Research in the USA, the UK Medical Research Council and the Departments of Health in the UK who increasingly require the submission of systematic reviews prior to agreeing new funding or taking strategic decisions. As these funders come under increasing pressure to be more accountable for the research pounds, euros or dollars spent, it is helpful to demonstrate, through the dissemination of evidence from systematic reviews, that they are not duplicating others' work inadvertently, but are funding research into identified gaps in knowledge, or building explicitly on existing research evidence. 


\section{Dental industry}

In recent years, the international manufacturers of toothpaste have provided an excellent example of EBD dissemination. They have not only undertaken and published a wide range of high-quality, randomised, controlled trials of cariespreventive and gum-health products (and thus made major contributions to the evidence base), but they have also raised awareness at the professional and public level through the active marketing of information on dental prevention and new findings from research. The manufacturers of dental materials also have a pivotal role to play in developing products to improve patient care and in some cases have taken a pro-active stance in dissemination. There are, however, some tensions where in vivo practice-based studies of materials may only become available once a product has been on the market for some time. There are significant opportunities here for technological progress to improve oral health and dental care, and for the more efficient and responsible communication of results to both dentists and their patients.

\section{Care providers and funders}

The individual care provider is very important: it is the clinician who is at the end of the EBD process. This aspect will be considered more in Part 3 of this series. The provider organisation whether this be part of a state-run health service, a health maintenance organisation or, in some countries, a dental body or business - should also have a strategic interest in EBD. Such organisations need to ensure that they are delivering clinically effective care based on current research evidence if they are to satisfy developing public and professional expectations and maintain activity in a competitive environment. Similarly, those who fund dental care, be they governments, insurance companies or patients, also have a developing and continuing interest in defining what constitutes the most appropriate and effective dental care for the location where they provide dental care, as well as in understanding how this should evolve in the light of new research findings. The UK Department of Health publication Options for Change $e^{5}$ is a good example that explicitly recognises the importance of using best evidence. ${ }^{6}$ This means using clinically relevant evidence where we have it, but acknowledging that there are a number of serious gaps in the evidence base supporting routine dental care.

Care providers and funders should be both the target for the dissemination of research findings and the active seekers of summaries of the findings of new, clinically relevant, research. In general, this is not yet the case.

\section{Professional associations}

Many professional associations seek to serve patients as well as dentists and have a similar range of interests as those outlined above. By exercising their corporate resources and influence, these associations have a huge potential to make a positive impact and secure beneficial changes in clinical practice. This potential might be developed in a number of ways, ranging from, for example, support for EBD educational initiatives, to partnership with dentalcare funders in professionally led strategic reform of dental practice for the future. Many elements of this large and heterogeneous group are not yet fully aware of all the complementary aspects of EBD. In order to achieve broader participation and support, it will be necessary to demystify the area and reduce the reliance on specialist jargon. There is also an urgent need to separate out the inevitably contentious issues around negotiations on remuneration and conditions of service from the need for professional leadership and securing support for a meaningful increase in high-quality dental research which is directly relevant to clinical practice. The American Dental Association's recent initiatives on EBD are a very encouraging sign that progress can be made.

\section{Undergraduate, postgraduate and continuing dental education}

Historically, there are close links between dental education and dental research, via institutions, people and funding. These links must be preserved despite tensions found in some countries that are eroding the number of dental teaching establishments. Teachers should still have efficient and regular links with researchers and be exposed research findings (both positive and negative results) in order to teach ethically and effectively. Undergraduate education must inculcate the dentists of tomorrow with an under- standing of both basic and applied science, the management of uncertainty and the development of new knowledge. Then, the graduate dentist needs to be willing to update and change clinical procedures over his/her lifetime of clinical practice. Critical-appraisal training and the attainment of modern information management skills should also be educational requirements.

Vocational training and postgraduate education can then build on this foundation and hone the skills of critical appraisal, systematic literature review and evidence-based clinical practice. Postgraduate study should also equip students with training that can cope with and contribute to an evolving and improving future in delivering clinical care. This is an area that could benefit from expansion of training opportunities with the modernisation of medical and dental education. Continuing professional development also provides ideal opportunities for the critical review of new knowledge. The challenge is to ensure that dentists participating in courses and related activities are open to new knowledge, and are prepared to contemplate changing clinical practice. Ideally, education providers should be actively involved in research, both to keep them up to date and to build credibility for this style of practice with students.

\section{Acknowledgement and disclaimer}

The author acknowledges financial support from the UK Department of Health and in particular, the Chief Scientist Office of the Scottish Executive Department of Health. The views expressed above are those of the author and do not necessarily represent the views of any health department.

1. Pitts NB. Understanding the jigsaw of evidencebased dentistry. 1. Research and synthesis. Evidence-Based Dent 2004; 5:2-4.

2. Getting evidence into practice Effective Health Care Bulletin. NHS Centre for Reviews and Dissemination, 1999; 5:1-16.

3. Clarkson JE, Pitts NB, Richards D, Shaw W, Worthington $\mathrm{H}$. Virtual Centre for Improving Oral Health. Evidence-Based Dent 2004; 5:5-6.

4. Pitts NB. Are we ready to move from operative to non-operative/preventive treatment of dental caries in clinical practice?. Caries Res 2004; 38:294-304.

5. Department of Health. NHS Dentistry: Options fo Change. London: Department of Health; 2002 (see www.doh.gov.uk/cdo/optionsforchange).

6. Pitts NB. NHS Dentistry: options for change in context. A personal overview of landmark document and what it could mean for the future of dental services. Br Dent J 2003; 195:631-635. 